motion Maxwell's law of distribution of energy would fail, assuming that law to consist in the ultimate equality of the energy of different parts of the system. He has thus shown the necessity for more accurate language than is commonly employed in the enunciation of that law, and a consideration of his prob. lem may help to determine the limits to which it is subject.

The following statement, whether co-extensive with Maxwell's law or not, will probably be accepted as true as far as it goes-

If there exist a very great number of material systems, the state of each being defined by certain co-ordinates and momenta, and if at a given instant all combinations of the co-ordinates and momenta are represented among them with frequency proportional to $\epsilon-h(x+T)$, then that distribution will be permanentthat is, will not be disturted by the mutual action of the systems, or by any forces in the field of which they are placed, provided all the forces concerned be conservative.

The further question as to how far the solution thus found for the permanent state is unique, has been treated by Boltzmann. He shows that a certain function, which in stationary motion must be positive and constant, necessarily diminishes with the time, so long as any small deviations exist from the above described state. It is obvious that this proposition of Boltzmann's cannot be applicable to all cases of stationary motion. Periodic motions are exceptions, and so is the system described by Lord Kelvin. The question is what assumptions underlie Boltzmann's demonstration. It will be of great advantage if one speaking with Lord Kelvin's authority will assist in defining the limits to which the proposition is subject.

Maxwell, although he may at times have expressed himself incautiously, was aware that the theory was subject to limita tions. The statistical, as distinguished from the historical, method was from his point of view of the essence of the theory. A distinction may be drawn between systems, such as Lord Kelvin's, to which the statistical method is inapplicable, and those in which the stationary motion, when attained, is what is called thermal motion-that is, the relative motions are in all directions indifferently, and of that irregular character in which heat is supposed to consist.

It may be that we shall be driven to the conclusion that Maxwell's law has no application except to this class of systems ; that it is, in fact, only the limiting state to which a material system approaches as we increase indefinitely the number of its degrees of freedom.

It does, at all events, appear that in cases where the law fails, its failure is due to the introduction of some restrictions on freedom of motion, especially as regards direction. Maxwell pointed out that dæemons-or, shall we say, beings endowed with free will-might by directing the courses of individual molecules cause a system to violate, not only the law of distribution of energy, but even the second law of thermodynamics. What these beings might be suppo:ed to do, that Lord Kelvin in fact does once for all for his system, by prescribing a priori the directions of motion and other conditions of the problem to suit his purpose.

H. W. WATSON.

S. H. BURBURY.

\section{The Former Connection of Southern Continents.}

WITH reference to the very interesting question treated in $\mathrm{Mr}$. Mellard Reade's letter of your issue of May 26 (p. 77), as to the former connection of southern continents, it may be worth while calling attention to the fact that a great circle, which I may call the Kaffraria Great Circle, connects that coast line with the Falkland Island and the South Georgia Island. It may be presumed that these two islands are the remaining summits of what was once a chain of mountains in connection with the continent of South America. Some of the points through which or near which this great circle passes are as follow - the abovementioned islands, Port de Sta. Cruz, Patagonia; it traverses the Pacific, runs parallel to the southern branch of the Aleutian Islands, and cuts Kamtchatka somewhat south of Klienchewskaia Volcano, and traversing Asia emerges by the Island of Cutch, so interesting on account of the earthquakes which occurred there. It is of interest to note that South Georgia Island is antipodal to the northern extremity of Saghalian Island.

Royal College of Science for Ireland, J. P. O'Reilly. Stephen's Green, Dublin, May 30.

No. I I 79 , vOL. 46]

\section{ON THE RELATIVE DENSITIES OF} HYDROGEN AND OXYGEN. ${ }^{1}$

I $\mathrm{N}$ a preliminary notice upon this subject (Roy. Soc. Proc., vol, xliii. p. 356 , February I 888 ), I explained the procedure by which 1 found as the ratio of densities $15^{\circ} 884$. The hydrogen was prepared from zinc and sulphuric, or from zinc and hydrochloric, acid, and was liberated upon a platinum plate, the generator being in fact a Smee cell, inclosed in a vessel capable of sustaining a vacuum, and set in action by closing the electric circuit at an external contact. The hydrogen thus prepared was purified by corrosive sublimate and potash, and desiccated by passage through a long tube packed with phosphoric anhydride. The oxygen was from chlorate of potash, or from mixed chlorates of potash and soda.

In a subsequent paper "On the Composition of Water" (Roy. Soc. Proc., vol. xlv. p. 425, February 1889), I attacked the problem by a direct synthesis of water from weighed quantities of the two component gases. The ratio of atomic weights thus obtained was 15.89 .

At the time when these researches were commenced, the latest work bearing upon the subject dated from i 845 , and the number then accepted was $15^{\circ} 96$. There was, however, nothing to show that the true ratio really deviated from the $16: 1$ of Prout's law, and the main object of my work was to ascertain whether or not such deviation existed. About the year r888, however, a revival of interest in this question manifested itself, especially in the United States, and several results of importance have been published. Thus, Prof. Cooke and Mr. T. W. Richards found a number which, when corrected for an error of weighing that had at first been overlooked, became I 5.869 .

The substantial agreement of this number with those obtained by myself, seemed at first to settle the question, but almost immediately afterwards there appeared an account of a research by Mr. Keiser, who used a method presenting some excellent features, and whose result was as high as $15^{\circ} 949$. The discrepancy has not been fully explained, but subsequent numbers agree more nearly with the lower value. Thus, Noyes obtains 15.896 , and Dittmar and Henderson give 15.866 .

I had intended further to elaborate and extend my observations on the synthesis of water from weighed quantities of oxygen and hydrogen, but the publication of Prof. E. W. Morley's masterly researches upon the "Volumetric Composition of Water" (Amer. Fourn. Sci., March 1891) led me to the conclusion that the best contribution that I could now make to the subject would be by the further determination of the relative densities of the two gases. The combination of this with the number $2.0002,{ }^{2}$ obtained by Morley as the mean of astonishingly concordant individual experiments, would give a better result for the atomic weights than any I could hope to obtain directly.

In the present work two objects have been especially kept in view. The first is simplicity upon the chemical side, and the second the use of materials in such a form that the elimination of impurities goes forward in the normal working of the process. When, as in the former determinations, the hydrogen is made from zinc, any impurity which that material may contain and communicate to the gas cannot be eliminated from the generator; for each experiment brings into play a fresh quantity of zinc, I "On the Relative Densities of Hydrogen and Oxygen. .II." Abstract of a paper by Lord Rayleigh, Sec.R.S., read at the Royal Society on February $18, \mathbf{1} \& q_{2}$.

lt should not be overlo ked that this number is difficult to reconcile with views generally held as to the applicability of Avogadro's law to very rare gases. From what we know of the behaviour of oxygen and hydrogen gases under compression, it seems improbable that volumes which are as $20002:$ under atmospheric conditions would remain as $2:$ I upon indefinite expansion. According to the formula of Van der Waals, a greater change than this in the ratio of volumes is to be expected. 
with its accompanying contamination. Moreover, the supply of acid that can be included in one charge of the generator is inadequate, and good results are only obtained as the charge is becoming exhausted. These difficulties are avoided when zinc is discarded. The only material consumed during the experiments is then the water, of which a large quantity can be included from the first. On the other hand, the hydrogen liberated is necessarily contaminated with oxygen, and this must be removed by copper contained in a red-hot tube. In the experiments to be described the generator was charged with potash, ${ }^{1}$ and the gases were liberated at platinum electrodes. In the case of a hydrogen filling, the oxygen blew off on one side from a mercury seal, and on the other the hydrogen was conveyed through hot tubes containing copper. The bulk of the aqueous vapour was deposited in a small flask containing strong solution of potash, and the gas then passed over solid potash to a long tube packed with phosphoric anhydride. Of this only a very short length showed signs of being affected at the close of all operations.

With respect to impurities, other than oxygen and oxides of hydrogen, which may contaminate the gas, we have the following alternative. Either the impurity is evolved much more rapidly than in proportion to the consumption of water in the generator, or it is not. If the rate of evolution of the impurity, reckoned as a fraction of the quantity originally present, is not much more rapid than the correspondingly reckoned consumption of water, the presence of the impurity will be of little importance. If, on the other hand, as is probable, the rate of evolution is much more rapid than the consumption of water, the impurity is soon eliminated from the residue, and the gas subsequently generated becomes practically pure. A similar argument holds good if the source of the impurity be in the copper, or even in the phosphoric anhydride; and it applies with increased force when at the close of one set of operations the generator is replenished by the mere addition of water. It is, however, here assumed that the apparatus itself is perfectly tight.

Except for the reversal of the electric current, the action of the apparatus is almost the same whether oxygen or hydrogen is to be collected. In the latter case the copper in the hot tubes is in the reduced, and in the former case in the oxidized, state. For the sake of dis. tinctness we will suppose that the globe is to be filled with hydrogen.

The generator itself is of the U-form, with unusually long branches, and it is supplied from Grove cells with about 3 amperes of electric current. Since on one side the oxygen blows off into the air, the pressure in the generator is always nearly atmospheric. Some trouble has been caused by leakage between the platinum electrodes and the glass. In the later experiments to be here recorded these joints were drowned with mercury. On leaving the generator the hydrogen traverses a red-hot tube of hard glass charged with copper, ${ }^{2}$ then a flask containing a strong solution of potash, and afterwards a second similar hot tube. The additional tube was introduced with the idea that the action of the hot copper in promoting the union of the hydrogen with its oxygen contamination might be more complete after removal of the greater part of the oxygen, whether in the combined or in the uncombined state. From this point onward the gas was nearly dry. In the earlier experiments the junctions of the hard furnace tubes with the soft glass of the remainder of the apparatus were effected by fusion. One of these joints remained in use, but the others were replaced by india-rubber connexions droiened in

t At the suggestion of Prof. Morley, the solution was freed from carbonate or nearly so, by the use of baryta, of which it contained a slight excess.

The copper must be free from sulphur; otherwise the contamination with sulphuretted hydrogen is somewhat persistent.

No, I I 79, VOL. 467 mercury. It is believed that no leakage occurred at these joints; but as an additional security a tap was provided between the generator and the furnace, and was kept closed whenever there was no forward current of hydrogen. In this way the liquid in the generator would be protected from any possible infiltration of nitrogen. Any that might find its way into the furnace tubes could easily be removed before the commencement of a filling.

Almost immediately upon leaving the furnace tubes the gas arrives at a tap which for distinctness may be called the regulator. In the generator and in the furnace tubes the pressure must be nearly atmospheric, but in the globe there is (at the commencement) a vacuum. The transition from the one pressure to the other takes place at the regulator, which must be so adjusted that the flow through it is approximately equal to the production of gas. At first the manipulation of the regulator was a source of trouble, and required almost constant attention, but a very simple addition gave the desired control. This was merely a long wooden arm, attached to the plug,which served both as a lever and as an indicator. Underneath the pointed extremity was a small table to which its motions could be referred. During the first two-thirds of a filling very little readjustment was needed, and the apparatus could be left for half an hour with but little fear of displacing too much the liquid in the generator. Towards the close, as the motive force fell off, the tap required to be opened more widely. Sometimes the recovery of level could be more conveniently effected by insertion of resistance into the electric circuit, or by interrupting it altogether for a few minutes. Into details of this kind it is hardly necessary to go further.

From the regulator the gas passed to the desiccating tubes. The first of these was charged with fragments of solid potash, and the second with a long length of phosphoric anhydride. Finally, a tube stuffed with glass wool intercepted any suspended matter that might have been carried forward.

The connection of the globe with the generator, with the Töppler, and with the blow-off, is shown in the accompanying figure. On the morning of a projected

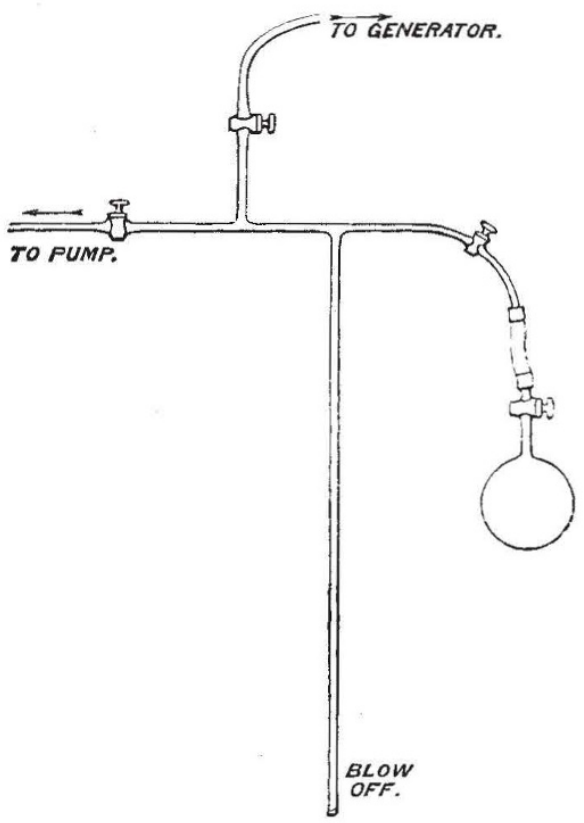

filling the vacuous globe would be connected with the free end of the stout-walled india-rubber tube, and secured by winding wire. The generator being cut off, a high vacuum would be made up to the tap of the globe. 
After a couple of hours' standing the leakage through the india-rubber and at the joints could be measured. The amount of the leakage found in the first two hours was usually negligible, considered as an addition to a globeful of hydrogen, and the leakage that would occur in the hours following would (in the absence of accidents) be still smaller. If the test were satisfactory, the filling would proceed as follows :--

The electric current through the generator being established, and the furnace being heated, any oxygen that might have percolated into the drying tubes had first to be washed out. In order to do this more effectively, a moderate vacuum (of pressure equal to about I inch of mercury) was maintained in the tubes and up to the regulator by the action of the pump. In this way the current of gas is made very rapid, and the half-hour allowed must have been more than sufficient for the purpose. The generator was then temporarily cut off, and a high vacuum produced in the globe connection and in the blow-off tube, which, being out of the main current of gas, might be supposed to harbour impurities. After this the pump would be cut off, the connection with the generator re-established, and, finally, the tap of the globe cautiously opened.

The operation of filling usually occupied from two to three hours. When the gas began to blow off under an excess of pressure represented by about half an inch of mercury, the blow-off cistern was lowered so as to leave the extremity of the tube free. For two minutes the current of gas from the generator was allowed to flow through, after which the generator was cut off, and the globe left in simple communication with the atmosphere, until it was supposed that equilibrium of pressure had been sufficiently established. Doubts have at various times been felt as to the interval required for this purpose. If too little time is allowed, there will remain an excess of pressure in the globe, and the calculated weight of the filling will come out too high. On the other hand, an undue prolongation of the time might lead to a diffusion of air back into the globe. In a special experiment no abnormal weight was detected after half an hour's communication, so that the danger on this side appeared to be small. When the passages through the taps were free from grease, one or two minutes sufficed for the establishment of equilibrium, but there was always a possibility of a partial obstruction. In the results to be presently given, four minutes were allowed after the separation from the generator. It may be remarked that a part of any minute error that may arise from this source will be eliminated in the comparison with oxygen, which was collected under like conditions.

The reading of the barometers and thermometers at the moment when the tap of the globe was turned off took place as described in the former paper. The arrangements for the weighings were also the same.

In the evacuations the process was always continued until, as tested by the gauge of the Töppler after at least a quarter of an hour's standing, the residue could be neglected. Here, again, any minute error would be eliminated in the comparison of the two gases.

In the case of oxygen, the errors due to contamination (even with hydrogen) are very much diminished, and similar errors of weighing tell very much less upon the proportional agreement of the final numbers. A comparison of the actual results with the two kinds of gas does not, however, show so great an advantage on the side of the oxygen as might have been expected. The inference appears to be that the individual results are somewhat largely affected by temperature errors. Two thermometers were, indeed, used (on opposite sides) within the wooden box by which the globe is surrounded, and they could easily be read to within $\frac{1}{20}{ }^{\circ} \mathrm{C}$. But in other respects, the circumstances were unfavourable, in consequence of the presence in the same room of the fur-

No, I 179 , vOL. 46] nace necessary to heat the copper. An error of $\pm \mathrm{O}^{\circ}$. I C. in the temperature leads to a discrepancy of I part in I 500 in the final numbers. Some further elaboration of the screening arrangements actually employed would have been an improvement, but inasmuch as the circumstances were precisely the same for the two gases, no systematic error can here arise. The thermometers were, of course, the same in the two cases.

The experiments are grouped in five sets, two for oxygen and three for hydrogen. In each set the work was usually continued until the tap of the globe required re-greasing, or until, owing to a breakage or to some other accident, operations had to be suspended.

The means are as follow :-

HYDROGEN.

\begin{tabular}{|c|c|c|c|c|}
\hline x89r. & Weight. & $\begin{array}{c}\text { Bar. } \\
\text { temp., F. }\end{array}$ & $\begin{array}{c}\text { Globe } \\
\text { temp., C. }\end{array}$ & $\begin{array}{l}\text { Corrected } \\
\text { to } 12^{\circ} \text {. }\end{array}$ \\
\hline 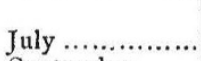 & $\begin{array}{c}\text { gram. } \\
0.15808\end{array}$ & 65 & is & 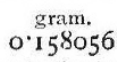 \\
\hline September ....... & 0.15797 & $6 \mathrm{I}$ & 17 & 0.157950 \\
\hline October ........... & 0.15804 & 53 & 12 & 0.158040 \\
\hline Mean ....... & & 60 & I6 & 0.158015 \\
\hline
\end{tabular}

OXYGEN.

\begin{tabular}{r|c|c|c|c}
\hline r89r. & Weight. & $\begin{array}{c}\text { Bar. } \\
\text { temp., F. }\end{array}$ & $\begin{array}{c}\text { Globe } \\
\text { temp., C. }\end{array}$ & $\begin{array}{c}\text { Corrected } \\
\text { to } 12^{\circ} .\end{array}$ \\
\hline June.............. & $\begin{array}{c}\text { grams. } \\
2.51785\end{array}$ & 68 & 0 & grams. \\
November ...... & 2.51720 & 55 & 13 & 2.51735 \\
\hline Mean ...... & & $6 \mathrm{r} \frac{1}{2}$ & $\mathbf{1} 6 \frac{1}{2}$ & 2.51713 \\
\hline
\end{tabular}

The means here exhibited give the weights of the two gases as they would be found with the globe at $12^{\circ} \mathrm{C}$., and the barometers at $60^{\circ} \mathrm{F}$. and at 30 inches. The close agreement of the mean temperatures for the two gases shows how little room there is for systematic error dependent upon imperfections in the barometers and thermometers. But the results still require modification before they can be compared with the view of deducing the relative densities of the gases.

In the first place, there is a systematic, though minute, difference in the pressures hitherto considered as corresponding. The terminal of the blow-off tube is 33 inches below the centre of the globe at the time of filling. In the one case this is occupied by hydrogen, and in the other by oxygen. If we treat the latter as the standard, we must regard the hydrogen fillings as taking place under an excess of pressure equal to $\frac{15}{16}$ of the weight of a column of oxygen 33 inches high; and this must be compared with 30 inches of mercury. Hence, if we take the sp. gr. of oxygen under atmospheric conditions at. 0.0014 , and that of mercury at 13.6 , the excess of pressure under which the hydrogen was collected is as a fraction of the whole pressure

$$
33 \cdot \frac{15}{16} \cdot \frac{0.0014}{13 \cdot 6}=0.000106 \text {; }
$$

and $0.000106 \times 0.158=0.000017$. This, then, is what we must subtract from the weight of the hydrogen on account of the difference of pressures due to the gas in the blowoff tube. Thus

$$
\mathrm{H}=0.157998, \quad \mathrm{O}=2.51724 .
$$

But there is still another and a more important correction to be introduced. In my former paper it was shown that when the weighings are conducted in air the true weight of the gas contained in the globe is not given 
by merely subtracting the weight of the globe when empty from the weight when full. When the globe is empty, its external volume is less than when full, and thus, in order to obtain the true weight, the apparent weight of the gas must be increased by the weight of air whose volume is equal to the change of volume of the globe.

In order to determine the amount of this change of volume, the globe is filled to the neck with recently boiled distilled water, and the effect is observed upon the level in the stem due to a suction of, say, 20 inches of mercury. It is not advisable to carry the exhaustion much further, for fear of approaching too nearly the point at which bubbles of vapour may be formed internally. In the earlier experiments, described in the preliminary note, the upper surface of the liquid was in the stem of the globe itself (below the tap), and the only difficulty lay in the accurate estimation of a change of volume occurring in a wide and somewhat irregular tube. The method employed was to produce, by introduction of a weighed quantity of mercury, a rise of level equal to that caused by the suction.

The advantage of this procedure lay in the avoidance of joints and of the tap itself, but, for the reasons given, the readings were not quite so accurate as might be desired. I wished, therefore, to supplement, if possible, the former determination by one in which the change of volume occurred in a tube narrower and of better shape. With this object in view, the stem of the globe was prolonged by a graduated tubular pipette attached with the aid of india-rubber. The tubes themselves were treated with gutta-percha cement, and brought almost into contact. It had hardly been expected that the joint would prove unyielding under the applied suction, but it was considered that the amount of the yielding could be estimated and allowed for by operations conducted with tap closed. The event, however, proved that the yielding at the joint was scarcely, if at all, perceptible.

The pipette, of bore such that $16 \mathrm{~cm}$. corresponded to I c.c., was graduated to $0^{\circ}$ I , and was read by estimation to $0^{\circ} 001$ c.c. In order the better to eliminate the changes due to temperature, readings under atmospheric pressure, and under a suction of 20 inches of mercury, were alternated. On January 28,1892 , a first set gave $0.648-$ $0^{\prime} 300=0^{\prime} 348$; a second gaveo $6645-0.316=0.3485$; and a third gave $0.675-0^{\circ} 326=0.349$. Similar operations with tap closed ${ }^{1}$ gave no visible movement.

The result of the day's experiments was thus 0.3485 for 20 inches, or 0.523 for 30 inches, suction. Similar experiments on January 28 , at a different part of the graduation, gave 0.526 . On this day the yielding with tap closed was just visible, and was estimated at o.0or. As a mean result, we may adopt 0.524 c.c. The graduation of the pipette was subsequently verified by weighing a thread of mercury that occupied a measured length.

A part of the above-measured volume is due to the expansion of the water when the pressure is relieved. We may take this at $0^{\circ} 000047$ of the volume per atmosphere. The volume itself may be derived with sufficient accuracy for the present purpose from the weight of its oxygen contents. It is $2.517 / 0^{\circ} 00137$, or 1837 c.c. The expansion of the water per atmosphere is thus $0.000047 \times 1837$, or 0.087 c.c. This is to be subtracted from 0.524 , and leaves 0437 c.c. This number applies strictly to the volume inclosed within the glass, but the change in the external volume of the globe will be almost the same.

The correction now under consideration is thus the weight of 0.437 c.c. of air at the average temperature of the balance room. The density of this air may be estimated at 0.00122 ; so that the weight of 0.437 c.c. is 0.000533 gram. This is the quantity which must be added to the apparent weights of the gases. The former

${ }^{1}$ For greater security the tap was turned while the interior was under suction.

NO. II79, VOL. 46] estimate was 0.00056 gram. The finally corrected weights are thus-

$$
\mathrm{H}=0.158531, \quad \mathrm{O}=2 \cdot 51777 \text {; }
$$

and for the ratio of densities we have

\subsection{2.}

This corresponds to a mean atmospheric condition of pressure and temperature.

If we combine the above ratio of densities with Prof. Morley's ratio of volumes, viz. 2.0002: 1 , we get, as the ratio of atomic weights, 15.880 .

If we refer to the table, we see that the agreement of the first and third series of hydrogen weighings is very good, but that the mean from the second series is decidedly lighter. This may have been in part fortuitous, but it is scarcely probable that it was so altogether. Under the circumstances we can hardly reckon the accuracy of the final results as closer than $\frac{1}{3000}$.

The accompanying table of results, found by various experimenters, may be useful for comparison :-

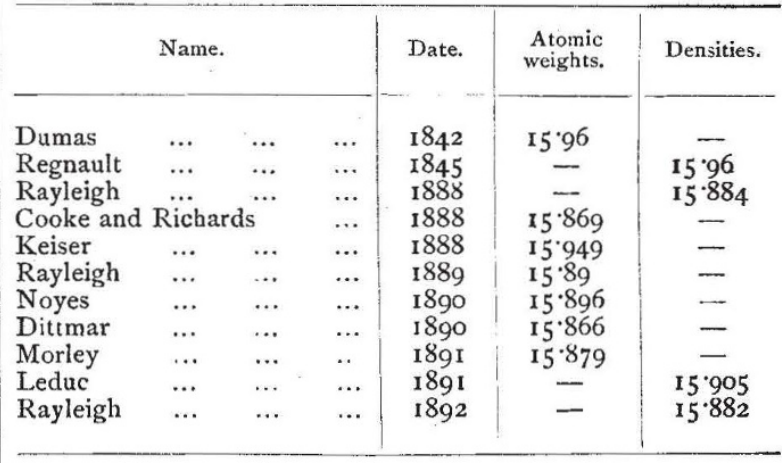

\section{THE ORIGIN OF THE YEAR.'}

II.

\section{Difficulties.}

THERE no doubt was a time when the Egyptian astronomer-priests imagined that, by the introduction of the 365 -days year, beginning at the solstice or the nearly contemporaneous Nile flood (there is an interval of three days between them in the present Coptic calendar ${ }^{2}$ ), and by marking the commencement, in addition, by the heliacal rising of one of the host of heaven, they had achieved finality. But alas! the dream must soon have vanished.

Even with this period of .365 days, the true length of the year had not been reached; and soon, whether by observations of the beginning of the inundation, or by observations of the solstice in some of the solar temples which, beyond all doubt, were then in existence, it was found that there was a difference of a day every four years between the beginning of the natural and of the newly-established year, arising, of course, from the fact that the true year is 365 days and a quarter of a day (roughly) in length.

The true year and this established year of 365 days, then, behaved to each other as follows. Let us take a year when the solstice, representing the beginning of the

- Continued from vol. xlv. p. 490

2 The calendar in question (given both by Brugsch and De Rougé) is, doubtless, a survival fro n old Egyptian times It is good for the neighbourhosd of Cairo, and the relation of the important days of the inundation to the solstice, in that part of the river, is as follows:-

\begin{tabular}{|c|c|c|c|c|c|}
\hline Night of the drop $\ldots \quad \ldots \quad \ldots$ & ... & rx Payni & & & \\
\hline Beginning of the inundation & ... & $\begin{array}{ll}\text { I5 } \\
\text { I8 }\end{array}$ & $\ldots$ & & $\begin{array}{l}\text { er solstice. } \\
\text { s after. }\end{array}$ \\
\hline Assembly at the Nilometer. & $\ldots$ & 25 & $\cdots$ & 10 &, \\
\hline Proclamation of the inundation & $\ldots$ & $26, ",$. & $\ldots$ & $1 \mathrm{I}$ & ,, \\
\hline Marriage of the Nile $\ldots \ldots$ & ... & 18 Mesori & $\cdots$ & 63 & " \\
\hline The Nile ceases to rise... $\quad .$. & ... & 16 Thoth & $\cdots$ & $9^{6}$ & , \\
\hline Opening of the dams $\ldots \ldots$ & $\cdots$ & I7 & $\cdots$ & 97 & , \\
\hline End of the greater inundation & ... & 7 Phaophi & $\cdots$ & II 7 & , \\
\hline
\end{tabular}

